\title{
Is the Use of Placebos Ethically Justified and are there Any Alternatives that can be of Equal Benefit in Advancing Current Medical Knowledge?
}

Togo A*

Specialty Registrar, Ninewells Hospital, Ninewells Avenue, Dundee, UK

*Corresponding author: Togo A, Specialty Registrar, Ninewells Hospital, Ninewells Avenue, Dundee, UK, Tel: +447880721732; E-mail: athena133@gmail.com

Received date: February 27, 2016; Accepted date: March 24, 2016; Published date: March 28, 2016

Copyright: () 2016 Togo A. This is an open-access article distributed under the terms of the Creative Commons Attribution License, which permits unrestricted use, distribution, and reproduction in any medium, provided the original author and source are credited.

\begin{abstract}
It is a well-established principle that, in order for a new drug to be proven effective, its therapeutic benefit to human subjects should be measured against that of either current established therapies, placebo, or, ideally, both. However, it can be argued that the use of placebo when alternative treatments are available contravenes the Declaration of Helsinki, as well as the Nuremberg Code which preceded it. This article puts forward arguments in favour of the use of placebos, and the ethical justifications behind this approach, that may be used by researchers when considering the design of a new trial.
\end{abstract}

Keywords: Placebo; Human subjects; Clinical trials; Nuremberg code

\section{Introduction}

The use of placebos in clinical trials has been an ethically contentious point, necessitating clarification in the Declaration of Helsinki [1].

The benefits, risks, burdens and effectiveness of a new intervention must be tested against those of the best proven intervention(s), except in the following circumstances:

Where no proven intervention exists, the use of placebo, or no intervention, is acceptable; or Where for compelling and scientifically sound methodological reasons the use of any intervention less effective than the best proven one, the use of placebo, or no intervention is necessary to determine the efficacy or safety of an intervention and the patients who receive any intervention less effective than the best proven one, placebo, or no intervention will not be subject to additional risks of serious or irreversible harm as a result of not receiving the best proven intervention.

\section{Extreme care must be taken to avoid abuse of this option.}

The need for including the use of placebo in the Declaration stems from the perception that there are cases where use of placebo may result in depriving a patient of effective treatment to help alleviate their condition, or prevent future damage. Safeguards need to be in place to ensure that this is not the case as this will contravene the position stated in the Nuremberg Code [2], that 'the experiment should be so conducted as to avoid all unnecessary physical or mental suffering or injury'. I will go on to discuss situations where the use of placebos is imperative, and has resulted in furthering medical knowledge, even in the presence of what may at first light seem effective treatments, but also ones in which it does indeed seem unethical that placebos have been used in a trial, and surprising that they were allowed to proceed from an ethics review board perspective.

\section{Commentary}

Pharmaceutical companies have to spend a significant amount of resources in the development of new drugs, from the discovery of a new chemical, to the planning of phase 1 to phase 3 trials and ultimately the permission from the government drug approval authority to allow the new drug to be released into circulation. This process can take around 12 years [3], and cost in the region of \$300-400 million. Ultimately, their aim is to elicit approval for their drug to be released into the market, for example by the Food and Drug Administration (FDA) in the US, or the Medicines and Healthcare products Regulatory Agency (MHRA) in the UK. This may influence the way trials are conducted, as there is certainly financial pressure on the part of the pharmaceutical companies to show benefit of their product. The ethical obligations of the researcher conducting such a clinical trial and the financial aims of the company may therefore be at odds, and it is at this point of potential conflict of interest, that the clinical trial regulatory authorities, such as the MHRA and local research ethics committees (RECs) are called upon to maintain the standards of ethical practice with regards to the conduct of these trials.

Defining the term placebo is not without its difficulties. The Oxford English Dictionary defines it as " $A$ medicine or procedure prescribed for the psychological benefit to the patient rather than for any physiological effect."

However, what this definition does not take into account is that there will be occasions where the psychological effect may lead to a measurable physiological effect, indeed it is this latter phenomenon that has led to the placebo controlled randomised trial being treated as the 'gold standard' among scientific trials. It has long been established that nociception has a strong psychological element, which may lead the patient who is receiving a placebo treatment, namely an inert substance, to report improvement in pain. But, as I will discuss further, this may not be the only, or even the main reason that the use of placebos in clinical trials has become almost ubiquitous.

The arguments for the use of placebo are multiple and, on the whole, quite convincing. Firstly, they provide a reference point. By comparing the new treatment against placebo, the effects, as well as the 
side effects of the new treatment can be gauged against a "zero" reference point. It is arguable if this is necessary though, as the aim of a study on a new treatment is generally to demonstrate its efficacy against the current available best treatment and not against no treatment, assuming that there is already a known treatment for the condition. This however, may be more of an idealistic pursuit, as the numbers of patients required to show what may be a small benefit, or equivalence, to current treatment may be such that the trial would need to run for many years, and at prohibitive costs, compared with a trial designed with a placebo arm.

Having a placebo arm may help a study reach statistical significance, because when comparing an active new treatment versus placebo, the statistical effect may become more pronounced than when comparing against a treatment that is already known to be effective. This means that statistical significance could be achieved at a lower number, leading to the recruitment of less patients, whilst saving the pharmaceutical companies money. This can be seen as both a good and a bad thing: if the costs of developing a new drug soared even higher than they are at present, due to the need for larger trials, the development of new drugs may slow down, much to the detriment of humanity. On the other hand, if new drugs are being authorised for use on the basis of small, placebo controlled trials, which help them achieve statistical significance at smaller numbers and without direct comparison to current best treatment, then humanity may be shortchanged, through the expensive acquisition and use of new therapies that may well add nothing to the existing, and likely cheaper, available treatments. This is truly a double edged sword.

Use of placebo may prevent researchers from having to make difficult decisions with regards to the suitability of each of the available medications or treatments in terms of efficacy and side effect profile, all of which need to be individually tailored to each patient. Therefore if a drug is being tested in one group, the other group receiving "best current treatment" may well not be homogeneous, if the best treatment for each patient is to be used, hence making drawing any conclusions difficult. The use of placebo circumvents this issue.

The most compelling argument however is that current best treatment may prove to be ineffective or even harmful when compared with placebo. This may well be one of the key reasons why use of placebos is still deemed to be the 'gold standard' by the FDA and other organisations. Due to issues such as lack of assay sensitivity, defined as the ability of a study to distinguish between effective and ineffective therapy, as well as publication bias towards positive studies, a number of approved treatments may not be shown to be statistically superior to placebo, in fact there are examples where the effective treatment fared worse than placebo [4]. If no placebos were ever used again once an acceptable treatment was made available, then comparison of acceptable versus new therapy may show equivalence, but will never be in a position to reveal a possible false positive effect of both the new and the existing treatment. A perhaps more extreme example of this is the comparison between two non-steroidal anti-inflammatory drugs, rofecoxib (Vioxx) and naproxen. The Vioxx Gastrointestinal Outcomes Research (VIGOR) trial [5] demonstrated a fivefold increase in the cardiac risk profile of Vioxx compared with naproxen, but it was not obvious whether this equated to a higher risk with the drug per se, or a reduced relative risk using naproxen, which may have been explained pharmacologically due to the difference in their respective actions. It wasn't until the APPROVe study, which remained unpublished, that Merck announced the withdrawal of rofecoxib [6]. The increased relative risk of cardiovascular events in this study was noted due to the inclusion of a placebo arm, and became apparent in this study, despite the fact that previous placebo controlled trials of Vioxx had shown it to be safe and effective. Sometimes 'old knowledge' may be superseded by new information, and therefore current accepted treatments and their efficacy should not be taken as gospel in every situation.

In contrast to some of the points made above, the arguments against the use of placebos stem mostly from an ethical concern about the use of humans in clinical trials who may be denied available treatment. Although consent is of paramount importance in recruiting patients for these studies, and one may argue that so long as the patient understands that they may not receive active treatment and consents to be entered into the trial 3 anyway, there is no ethical issue. But using an example of the trials of new disease modifying medications (DMARDs) in rheumatoid arthritis [7], it is clear that accepting that some patients will receive placebo in this setting means that the researchers decide how much pain and disability the trial participants may come under, and thus doesn't correlate with the Nuremberg code, par 4, that "the experiment should be so conducted as to avoid all unnecessary physical or mental suffering or injury" [2]. Similarly in the paper by Rickels et al. [8] the introduction states that 'Effective antidepressant compounds have been available for over 30 years', yet in the trial paroxetine has been compared with placebo and not against any of the conventional available medications, resulting in a series of patients not being given any treatment for their condition. Is it really ethical to allow a group of patients to be treated with placebo, however significant its psychological effects may be in a group of patients with mental illness, instead of long standing pharmacological therapy that we know works?

The reality is that the pharmaceutical companies are under pressure to trial their new products against placebo, both for reasons of easier trial design and execution, as I mentioned earlier, but also due to the apparent stance of the drug approving authorities. The FDA is ambiguous about its advice regarding the use of placebos, and whilst on the one hand it suggests that new treatments should be tested against the existing ones, placebo controlled trials seem to be considered the gold standard, and examples of that are found both in the testing of new disease modifying agents for RA, as well as a study on the effects of atenolol which was rejected as there was no placebo arm [9]. In this instance, the FDA refused to approve the new drug, atenolol, a beta-blocker for use in angina pectoris, even though the application showed that it had an effect similar to that of propranolol, an already approved drug. The application was rejected because the drug had not been tested against placebo, even though a placebo controlled trial would have violated the Declaration of Helsinki.

So what could the best way forward with regards to the future of clinical trials, based on the points raised above? I think the local research ethics committees, as well as the drug approving authorities, such as the FDA in the US, and the Medicines and Healthcare Regulatory Authority (MHRA) in the UK, should convene on the matter and try to publish some guidelines for drug companies and researchers involved in developing new drugs, highlighting situations wherein the use of placebo would be deemed compulsory, and those where it would be preferable to use current treatment as the comparator. Some attempt at uniformity will help guide these trials, which are extremely costly, both from a human and a financial perspective, in order for conclusions to be drawn safely, yet ethically. Perhaps even more importantly, this should ensure that no trials have to be abandoned or their results invalidated secondary to technical 
considerations that may have arisen after the study had commenced, for example the aforementioned atenolol trial [9].

Situations where placebo would be compulsory may be ones where there is no existing treatment or perhaps those where the only current treatment has been found to have considerable side effects and as such is poorly tolerated, in which case it could be argued, always on an individual basis, that placebo may be more acceptable than current therapy. It may also be appropriate to use placebo in situations where trials involve patients who have already been on current best treatment and have stopped responding to it, which may be the case for some cancer therapies or immunological treatments for autoimmune disorders. The advantage of discontinuing treatment in this group and using placebo would be that the side effects of the current therapy are removed from the equation, while at the same time the patient is not deprived of life changing treatment. On the other hand, where effective therapies exist, particularly if they are well tolerated, the best practice would be to compare the new treatment against the 'old gold standard', thus comparing both effect and side effect profile directly, whilst maintaining an ethically acceptable practice.

As will have become evident from the arguments raised above, striking the right balance with regards to clinical trials and whether to use placebos or not is a difficult task for all involved. The easiest position is held by researchers who are trialling a new drug or method for a condition that has no current alternative, as in this case no treatment, or placebo prescription, is unarguably ethical as the patient is not deprived of known alternatives. If the drug does turn out to provide a significant benefit, one may argue in hindsight that those who received the active drug were better served, but employing the principle of equipoise at the time that the trial began should silence any later criticism. With regards to therapeutic trials for which there is an existing alternative, the use of placebo should be tightly controlled by local and national ethics committees that are monitoring all research. This may involve grading the quality of evidence and strength of recommendations on any treatment according to a framework, as proposed by Atkins et al [10]. This study provides a platform to allow a structured consideration of the balance between risk and benefit, the quality of the evidence and its applicability in certain situations and the likelihood of baseline risks. It can be argued that only when the patients have undergone such grading by the researchers, and on balance it is decided that placebo is better or at least no less beneficial than other current recognised treatments, that the patients should be placed in a placebo arm of a trial. Specific agreement on the matter and further guidance from national ethics committees as well as the drug approving authorities may be what is required in future to better approach this complex issue.

\section{References}

1. World Medical Association (2004) World Medical Association declaration of Helsinki: Ethical principles for medical research involving human subjects.

2. U.S. Department of Health \& Human Services (2005) The Nuremberg Code.

3. California Biomedical Research Association (2005) New Drug development process factsheet.

4. Khan A, Khan SR, Walens G, Kolts R, Giller EL (2003) Frequency of positive studies among fixed and flexible dose antidepressant clinical trials: An analysis of the Food and Drug Administration summary basis of approval reports. Neuropsychopharmacology 28: 552-557.

5. Bombardier C, Laine L, Reicin A, Shapiro D, Burgos-Vargas, et al. (2000) Comparison of upper gastrointestinal toxicity of rofecoxib and naproxen in patients with rheumatoid arthritis. N Engl J Med 343: 1520-1528.

6. Merck \& Co., Inc (2000) Merck announces the voluntary withdrawal of Vioxx.

7. Felson DT, Anderson JJ, Meenan RF (1990) The comparative efficacy and toxicity of second-line drugs in rheumatoid arthritis: results of two metaanalyses. Arthritis Rheum 33: 1449-1461.

8. Rickels K, Amsterdam J, Clary C, Fox I, Schweizer E, et al. (1992) The efficacy and safety of paroxetine compared with placebo in outpatients with major depression. J Clin Psychiatry 3: 30-32.

9. Temple R (1982) Government viewpoint of clinical trials. Drug Inf J16: 10-17.

10. Atkins D, Best D, Briss PA, Eccles M, Falck-Ytter, et al. (2004) Grading quality of evidence and strength of recommendations. BMJ 328:1490-1498. 